\title{
Fetal and Neonatal MRI Predictors of Aggressive Early Clinical Course in Vein of Galen Malformation
}

\author{
(D). Arko, (D) M. Lambrych, (D) A. Montaser, (D) Zurakowski, and (DD.B. Orbach
}

\begin{abstract}
BACKGROUND AND PURPOSE: Neonates with vein of Galen malformations are split into 2 cohorts: one needing urgent neonatal embolization, with relatively high mortality and morbidity even with expert care, and a cohort in which embolization can be deferred until infancy, with far better prognosis. We aimed to identify brain MR imaging characteristics obtained from fetal and early neonatal scans that can predict the clinical presentation.
\end{abstract}

MATERIALS AND METHODS: Patients with vein of Galen malformations were stratified into a neonatal at-risk cohort if the patient needed urgent neonatal intervention or if neonatal death occurred; or an infantile treatment cohort if they were stable enough not to require treatment until $>1$ month of age. Twelve vascular MR imaging parameters, measured by 2 independent observers, were systematically correlated with the need for early neonatal intervention and/or neonatal mortality.

RESULTS: A total of 32 neonatal patients (21 patients in the neonatal at-risk cohort, 11 in the infantile treatment cohort) were identified. Maximal mediolateral diameter (area under the curve $=0.866, P<.001$ ) and cross-sectional area (area under the curve $=0.836, P=.002$ ) at the narrowest point of the straight or falcine sinus were most predictive of clinical evolution into the neonatal at-risk cohort. There were 15 patients who had fetal MRIs (10 in the neonatal at-risk cohort and 5 in the infantile treatment cohort). Here too, maximal mediolateral diameter (area under the curve $=0.980, P=.003$ ) and cross-sectional area (area under the curve $=0.941, P=.007$ ) at the narrowest point of the straight or falcine sinus were highly predictive of the neonatal at-risk cohort.

CONCLUSIONS: Early neonatal and fetal MR imaging can be readily used for accurate early risk stratification, assisting in directing resources, timing treatment decisions, and identifying appropriate cohorts for novel interventions.

ABBREVIATIONS: AUC = area under the curve; BA-MD = basilar artery maximal diameter; $C C=$ craniocaudal diameter; ICA-MD = internal carotid artery maximal diameter; IT infantile treatment cohort; NAR = neonatal at-risk cohort; ROC = receiver operating characteristic; Sig-MD = sigmoid sinus maximal diameter; SS-A = straight sinus cross-sectional area at the narrowest point of the straight or falcine sinus; SS-MD = straight sinus maximal diameter at the narrowest point of the straight or falcine sinus; SS-P = straight sinus outer perimeter at the narrowest point of the straight or falcine sinus; VOGM = vein of Galen malformations

$\mathbf{V}$ ein of Galen malformations (VOGM) are rare congenital cerebral arteriovenous lesions that represent approximately $30 \%$ of all pediatric vascular anomalies. ${ }^{1}$ The condition is characterized by presumed persistence of direct arteriovenous communications between the embryonic choroidal arteries and the

Received March 5, 2020; accepted after revision April 3.

From the Departments of Neurosurgery (L.A., M.L., A.M., D.B.O.); Anesthesiology and Surgery (D.Z.); and Neurointerventional Radiology (D.B.O.), Boston Children's Hospital, Harvard Medical School, Boston, Massachusetts.

This work was supported by the Sage Schermerhorn Fund for Image-Guided Therapy.

Please address correspondence to Darren B. Orbach, MD, PhD,

Neurointerventional Radiology, Boston Children's Hospital, 300 Longwood Avenue Boston, MA 02115; e-mail: darren.orbach@childrens.harvard.edu

Indicates article with supplemental on-line photo.

http://dx.doi.org/10.3174/ajnr.A6585 median prosencephalic vein of Markowski. ${ }^{2,3}$ The latter normally involutes by 11 weeks' gestation.

The development of endovascular embolization techniques for VOGM in the 1990s transformed the condition from nearuniversal mortality or severe morbidity to a potentially manageable condition, as demonstrated by multiple institutional cohorts and 2 recent meta-analyses. ${ }^{4,5}$ However, mortality and severe neurocognitive morbidity remain daunting challenges for patients with vein of Galen malformations. A recent literature review of all published prenatally diagnosed cases reported a $54 \%$ mortality rate, a $14 \%$ rate of survival with severe neurocognitive compromise, and only an approximately one-third chance of survival without severe neurologic impairment. ${ }^{6}$ In this study, approximately two-thirds of patients with VOGM presented in the first few days of life with aggressive high- 

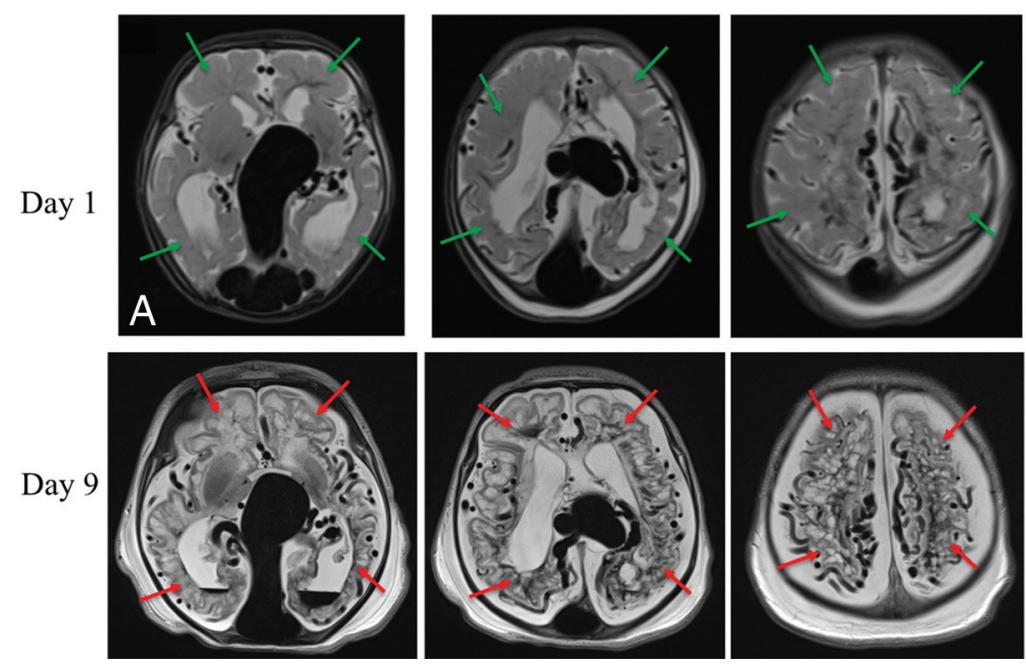

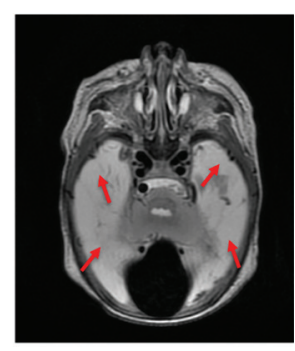

Day 1

FIG 1. Aggressive early evolution of a vein of Galen malformation (NAR cohort) (A, left panels). After med-flighting this neonate with VOGM to our center for urgent treatment, the MR imaging obtained on day-of-life 9 showed diffuse bihemispheric brain injuries (red arrows) not seen on the initial on day-of-life 1 MR imaging (green arrows showing analogous regions). Increased soft-tissue edema in the neck and scalp on day 0 is secondary to overwhelming heart failure ( $B$, right panels). Another patient, a one-day-old neonate with VOGM presents with complete liquefactive gliosis of both cerebral hemispheres (white brain signal resembles white CSF signal on T2-weighted imaging).

output cardiopulmonary failure, requiring several rounds of high-risk urgent embolization; we refer to these patients as the neonatal at-risk cohort (NAR). A report with nationwide population data from the United Kingdom reported, in this NAR cohort with aggressive neonatal presentation, a $40 \%$ mortality with $50 \%$ of the survivors having severe neurocognitive compromise and an overall $30 \%$ likelihood of survival to adulthood without significant neurologic injury. ${ }^{7}$ Several examples of the kind of global parenchyma brain injuries seen in such patients are shown in Fig 1.

Note that at our center, if diffuse parenchymal brain injury or hemorrhage is seen on fetal imaging, comfort care rather than embolization is recommended. Fortunately, such cases are extremely rare, because the brain parenchymal findings in most cases appear postnatally. However, such patients invariably manifest severe cardiopulmonary decompensation at birth and are thus included in the NAR cohort. The remaining approximately one-third of patients with VOGM present after the neonatal period, often with macrocephaly, ventriculomegaly, or seizures; we refer to these patients as the infantile treatment cohort (IT). These patients are typically embolized at several months of age, and the same UK national population data found a $90 \%$ survival rate in this more stable IT cohort. ${ }^{8}$ An example of the response to embolization in an infant with this presentation is shown in the On-line Figure.

Clinical presentation based on prenatal imaging has been difficult to predict, with no particular marker being found to correlate with a benign course. Thus, at most expert centers, including our own, every neonate with VOGM is admitted to the neonatal intensive care unit and carefully observed for clinical deterioration, with the team prepared for urgent embolization as needed. The wide range of possible outcomes, from normal neurocognitive development and life span to death within days or severe neurologic compromise, is unnerving to families and providers and stymies efforts to triage care. Thus, we first sought to determine whether vascular anatomic differences based on an early neonatal MR imaging measurement could differentiate the NAR and IT and thus aid in risk stratification. If so, as a second step, we sought to ascertain whether the same would hold for fetal MR imaging. Identification of prenatal and early neonatal predictors would allow advance preparation for the likely clinical evolution, offer potential guidance in parental consideration of possible termination of pregnancy, and allow identification of appropriate patient cohorts for the development of novel therapeutic approaches.

Finally, it has been hypothesized that 1 particular morphologic subtype of vein of Galen malformation, the choroidal subtype (consisting of multiple, often innumerable bilateral arteriovenous fistulas investing the walls of the prosencephalic varix), is associated with NAR compared with the mural subtype (consisting of a single arteriovenous fistula). Thus, along with anatomic measurements, we sought to determine whether, in fact, morphology was such a predictor.

\section{MATERIALS AND METHODS}

After obtaining approval of our institutional review board, a prospective data base of patients who presented with a VOGM from 2007 through 2018 was used to identify the cohort of interest. For the initial analysis, inclusion criteria consisted of all patients with VOGM who had brain MR imaging within the first 10 days of life, before the initiation of any treatment; all patients with only posttreatment scans available were excluded. Note that for nearly the entire neonatal cohort, the neonatal scan was obtained on day-of-life 1 after birth. For the fetal cohort, we included all patients with fetal MR images obtained as part of consultation at our Maternal Fetal Care Center where neonatal clinical follow-up was also available. Demographic information was collected. 

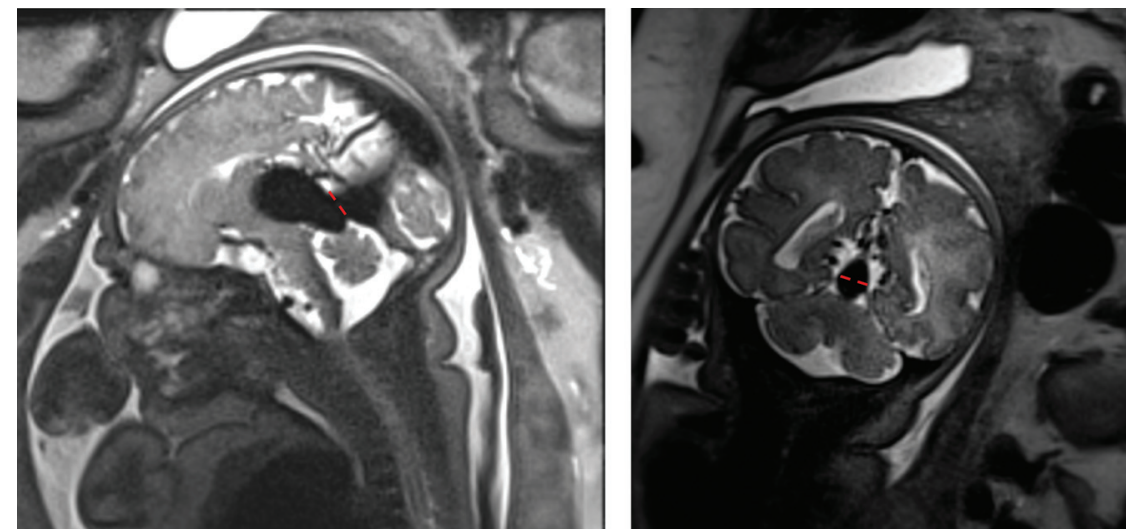

FIG 2. Measurement of the mediolateral diameter of the falcine sinus at its shortest point. The left image shows a sagittal view of a fetal MR imaging, with the dashed red line at the section of shortest height of the falcine sinus. The right image shows a coronal view with the mediolateral diameter of the sinus demonstrated with red dashes, measured at this same shortest section. This diameter efficiently differentiated the IT from the NAR cohort, as did the cross-sectional area of the sinus, measured at this same section.

All images were viewed using a PACS system by 2 independent readers, a neuroendovascular fellow (reader 1 ) and the senior author (reader 2). The readers were blinded to the patient's clinical outcome. Twelve cerebrovascular anatomic parameters were measured manually in the PACS in the entire neonatal cohort. Neonatal subjects for whom all measurements could not be obtained due to image quality and/or lack of relevant sequences were excluded from the study. Attempts were made to obtain all measurements in the fetal cohort as well, but given the variability intrinsic to fetal imaging secondary to fetal movements, fetal subjects were not excluded from the study if it was not possible to obtain all measurements. Parameters included the following: the maximal diameter of the prosencephalic varix (anterior-posterior, craniocaudal [CC], and mediolateral); maximal diameter of the basilar artery (BA-MD) and the internal carotid arteries bilaterally (ICA-MD); maximal diameter of the sigmoid sinuses (SigMD) bilaterally; and the maximal mediolateral diameter of the straight or falcine sinus at its narrowest point in the craniocaudal axis (ie, its shortest point, along the course from the prosencephalic varix to the torcular [SS-MD]) (Fig 2). This last measurement was designed to assess the degree of constraint on outflow by assessing the caliber of the point of maximal constriction.

In addition, as potentially even more precise assessments of flow constraint, the perimeter of the straight or falcine sinus at this narrowest point (SS-P) was also measured by reader 1, while reader 2 measured the cross-sectional area at this point (SS-A). Each malformation was independently categorized morphologically as mural or choroidal by reader 2 . In addition, the presence or absence of an occipital sinus was also noted (by both readers), because the nonregression of this fetal venous sinus might potentially correlate with markedly increased flow return to the systemic circulation.

Patients were categorized as either NAR, those who either continued to decline despite maximal medical management of their severe cardiopulmonary failure and who thus underwent urgent neonatal embolization as a potential life-saving tinuous variables. intervention or those who died before such intervention could be given; or as IT, those who were successfully managed medically in the neonatal intensive care unit, extubated, and discharged home. These patients underwent elective embolization in infancy (typically at 3-6 months of age).

\section{Statistical Analysis}

Univariate analysis comparing cohorts was conducted using the nonparametric Mann-Whitney $U$ test. Statistical significance was set at 2 -tailed $P<.01$, using the Holm test to account for multiple variables being tested to protect against type I false-positive errors. ${ }^{9}$ The MannWhitney $U$ test was calculated for both independent readers. To assess interrater reliability, we calculated a 2-way mixed intraclass correlation coefficient between readers for con-

For categoric variables, VOGM morphology, and occipital sinus presence, a Pearson $\chi^{2}$ analysis was used. Interrater chancecorrected reliability was measured by the Cohen $\kappa$. Imaging variables chosen for multivariable logistic regression analysis were based on a level of significance from univariate analysis.

On the basis of the measurements of reader 2, multivariable logistic regression using backward-selection stepwise modeling was used to identify independent imaging parameters that differentiate the NAR and IT cohorts and to determine the probability of NAR. For model selection, we chose a $P$ value criterion of $<.05$ for entry and a less stringent value of .10 for retaining a variable in the model to avoid missing important imaging parameters, and we obtained the same results with a backward and forward selection, which we interpreted as a sign of a robust multivariable model. A receiver operating characteristic (ROC) curve was created to assess differentiation of the 2 cohorts using SS-MD and SS-A with an area under the curve (AUC) to assess predictive accuracy. The Youden $\mathrm{J}$ index was used to identify the best cutoff value for SS-MD and SS-A, to maximize the combination of sensitivity and specificity.

Univariate and multivariable analyses were performed separately for the early neonatal and fetal MR image sets. Both a paired-samples $t$ test and a Pearson correlation $(r)$ coefficient were used to compare continuous variables of patients who had both fetal and corresponding early neonatal MR imaging. All statistical analyses were performed using SPSS statistical software, Version 25.0 (IBM).

\section{RESULTS}

In the early neonatal MR imaging cohort, there was a total of 32 patients, with 21 patients in the NAR cohort and 11 in the IT cohort. Mean gestational age was $38 \pm 2.3$ weeks, with no significant age difference between the NAR ( $37.7 \pm 2.8$ weeks) and IT (38.5 \pm 1.3 weeks) cohorts $(P=.453)$. Univariate analysis (Table 1$)$ 
Table 1: Fetal MR imaging scan univariate data

\begin{tabular}{|c|c|c|c|c|c|c|c|c|c|c|c|c|c|c|c|}
\hline \multirow[b]{3}{*}{ Region } & \multicolumn{7}{|c|}{ Reader 1} & \multicolumn{7}{|c|}{ Reader 2} & \multirow[b]{3}{*}{ ICC } \\
\hline & \multicolumn{3}{|c|}{ NAR } & \multicolumn{3}{|c|}{ Infant Treatment } & \multirow{2}{*}{$\begin{array}{c}P \\
\text { Value }\end{array}$} & \multicolumn{3}{|c|}{ NAR } & \multicolumn{3}{|c|}{ Infant Treatment } & \multirow{2}{*}{$\begin{array}{c}P \\
\text { Value }\end{array}$} & \\
\hline & No. & Median & IQR & No. & Median & IQR & & No. & Median & IQR & No. & Median & IQR & & \\
\hline $\begin{array}{c}\text { Varix ML } \\
(\mathrm{mm})\end{array}$ & 10 & 18 & $13-22$ & 4 & 19 & $11-25$ & 1.00 & 10 & 18 & $13-24$ & 5 & 20 & $12-23$ & 1.00 & 0.98 \\
\hline $\begin{array}{c}\text { Varix AP } \\
\text { (mm) }\end{array}$ & 10 & 30 & $28-33$ & 4 & 19 & $12-25$ & $<.01$ & 9 & 30 & $21-34$ & 5 & 20 & $16-27$ & .15 & 0.75 \\
\hline $\begin{array}{c}\text { Varix CC } \\
(\mathrm{mm})\end{array}$ & 10 & 20 & $18-21$ & 4 & 16 & $8-25$ & .64 & 10 & 18 & $12-21$ & 5 & 18 & $11-23$ & .77 & 0.89 \\
\hline $\begin{array}{l}\text { Varix V } \\
\qquad\left(\mathrm{CM}^{3}\right)\end{array}$ & 10 & 5 & $2-5$ & 4 & 3 & $1-5$ & .73 & 9 & 5 & $2-6$ & 5 & 5 & $2-6$ & 1.00 & 0.93 \\
\hline $\begin{array}{l}\text { SS-MD } \\
(\mathrm{mm})\end{array}$ & 10 & 10 & $8-12$ & 4 & 6 & $4-6$ & $<.01$ & 10 & 8 & $5-11$ & 5 & 3 & $3-5$ & $<.001$ & 0.95 \\
\hline SS-P (mm) & 9 & 43 & $39-49$ & 4 & 28 & $22-34$ & $<.001$ & 10 & 39 & $28-41$ & 5 & 20 & $17-31$ & $<.01$ & 0.87 \\
\hline $\begin{array}{l}\text { SS-A } \\
\left.\qquad \mathrm{mm}^{2}\right)\end{array}$ & & & & & & & & 10 & 85 & 56-112 & 5 & 20 & $18-45$ & $<.01$ & \\
\hline $\begin{array}{l}\text { BA-MD } \\
(\mathrm{mm})\end{array}$ & 5 & 3 & $3-4$ & 3 & 3 & $2-0$ & .57 & & & & & & & & \\
\hline $\begin{array}{l}\text { R ICA-MD } \\
\text { (mm) }\end{array}$ & 5 & 4 & $3-5$ & 3 & 3 & $2-0$ & .57 & & & & & & & & \\
\hline $\begin{array}{l}\text { L ICA-MD } \\
\text { (mm) }\end{array}$ & 5 & 3 & $3-5$ & 3 & 3 & $2-0$ & .39 & & & & & & & & \\
\hline $\begin{array}{l}\text { R Sig-MD } \\
(\mathrm{mm})\end{array}$ & 8 & 10 & $9-12$ & 4 & 6 & $4-8$ & $<.001$ & 10 & 7 & $6-8$ & 5 & 5 & $4-6$ & $<.001$ & 0.72 \\
\hline $\begin{array}{l}\text { L Sig-MD } \\
\text { (mm) }\end{array}$ & 8 & 11 & 7-12 & 4 & 6 & $5-7$ & .05 & 10 & 9 & $7-9$ & 5 & 6 & $4-6$ & $<.01$ & 0.94 \\
\hline
\end{tabular}

Note:-AP indicates anterior-posterior diameter; IQR, interquartile range; L, left; ML, mediolateral diameter; R, right; $\mathrm{V}$, volume; ICC, intraclass correlation coefficient. Statistical significance was set using a conservative criterion of $P<.01$ to account for multiple testing.

Table 2: Early MR imaging scan data for neonatal at-risk and infant treatment cohorts

\begin{tabular}{|c|c|c|c|c|c|c|c|c|c|c|c|c|c|c|c|}
\hline \multirow[b]{3}{*}{ Region } & \multicolumn{7}{|c|}{ Reader 1} & \multicolumn{7}{|c|}{ Reader 2} & \multirow[b]{3}{*}{ ICC } \\
\hline & \multicolumn{3}{|c|}{ NAR } & \multicolumn{3}{|c|}{ Infant Treatment } & \multirow[b]{2}{*}{$P$} & \multicolumn{3}{|c|}{ NAR } & \multicolumn{3}{|c|}{ Infant Treatment } & \multirow[b]{2}{*}{$P$} & \\
\hline & No. & Med & IQR & No. & Med & IQR & & No. & Med & IQR & No. & Med & IQR & & \\
\hline Varix ML (mm) & 21 & 24 & $18-27$ & 11 & 21 & $19-25$ & .46 & 21 & 23 & $17-27$ & 11 & 21 & $18-26$ & .97 & 0.921 \\
\hline Varix AP (mm) & 21 & 30 & $19-35$ & 11 & 20 & $20-27$ & .22 & 21 & 30 & $25-35$ & 11 & 22 & $20-29$ & .05 & 0.633 \\
\hline Varix CC (mm) & 20 & 24 & $20-27$ & 11 & 19 & $16-24$ & .16 & 21 & 21 & $16-29$ & 11 & 18 & $15-24$ & .14 & 0.875 \\
\hline Varix $\vee\left(\mathrm{CM}^{3}\right)^{\prime}$ & 21 & 6 & 4-10 & 11 & 4 & $3-8$ & .24 & 20 & 9 & $4-13$ & 11 & 4 & $3-8$ & .15 & 0.961 \\
\hline SS-MD (mm) & 17 & 10 & $8-14$ & 11 & 6 & $5-8$ & .00 & 21 & 9 & 7-11 & 11 & 3 & $2-6$ & $<.001$ & 0.983 \\
\hline SS-A $\left(\mathrm{mm}^{2}\right)$ & 21 & 6 & $4-8$ & 11 & 3 & $3-4$ & .00 & 20 & 85 & $65-123$ & 11 & 28 & $18-55$ & $<.001$ & \\
\hline SS-P (mm) & 17 & 40 & $36-48$ & 11 & 29 & $21-37$ & .00 & 20 & 35 & $32-43$ & 11 & 27 & $21-35$ & .03 & 0.969 \\
\hline BA-MD (mm) & 21 & 4 & $3-5$ & 11 & 4 & $3-4$ & .03 & 21 & 4 & $3-4$ & 11 & 3 & $2-3$ & $<.01$ & 0.877 \\
\hline R ICA-MD (mm) & 21 & 4 & $4-5$ & 11 & 3 & $2-4$ & .00 & 21 & 4 & $3-4$ & 11 & 3 & $2-3$ & $<.001$ & 0.851 \\
\hline L ICA-MD (mm) & 21 & 4 & $3-5$ & 11 & 3 & $3-3$ & .00 & 21 & 4 & $3-4$ & 11 & 3 & $2-3$ & $<.01$ & 0.655 \\
\hline R Sig-MD (mm) & 21 & 9 & $7-10$ & 11 & 7 & $4-9$ & .04 & 21 & 6 & $5-8$ & 11 & 5 & $2-6$ & .02 & 0.642 \\
\hline L Sig-MD (mm) & 21 & 8 & $8-11$ & 11 & 7 & $6-9$ & .10 & 21 & 7 & $6-8$ & 11 & 4 & $3-6$ & $<.001$ & 0.775 \\
\hline
\end{tabular}

Note:-Med indicates median; AP, anterior-posterior diameter; IQR, interquartile range; L, left; ML, mediolateral diameter; R, right; $V$, volume; ICC, intraclass correlation coefficient. Statistical significance was set using a conservative criterion of $P<.01$ to account for mutliple testing.

showed that varix caliber measurements (varix mediolateral diameter, varix anterior-posterior, varix CC), varix volume, and right SigMD were not significant between the 2 cohorts, as measured by both readers. The 2 readers differed in their assessment of whether the left Sig-MD, SS-P, and BA-MD measurements differed significantly. Significant differences between cohorts were found and were consistent between both readers for SS-MD, right ICA-MD, and left ICA-MD. Interrater reliability, as measured by the intraclass correlation coefficient, was $>0.80$ for most caliber measurements (varix mediolateral diameter, varix $\mathrm{CC}$, varix volume, SS$\mathrm{MD}$, SS-P, BA-MD, and right ICA-MD (Table 2).

VOGM morphology was of the choroidal type, in most cases for both readers. There was a significant difference in VOGM morphology between cohorts based on the observations of reader $2(P<.01)$, but this was not reproduced by reader 1 ; thus, the interrater reliability was moderate $(\kappa=0.48, P=.05)$ (Table 3$)$. The occipital sinus was present in all of the NAR cohort as observed by both readers, and its presence was significantly different between cohorts (Table 4). Thus, absence of an occipital sinus in a neonatal MR imaging strongly implies belonging to the IT cohort. The interrater reliability for observation of the occipital sinus was high $(\kappa=0.819, P<.001)$ (Table 4$)$.

After performing multivariable analysis using the measurements that significantly differed between the cohorts and had a high interrater reliability, the most predictive parameters were SS-MD $($ AUC $=0.866, P<.001)$ and SS-A $($ AUC $=0.836$, 
$P=.002$ ) (Table 5 and Fig $3 A$ ). ROC curves showed that an SSMD of $>6.2 \mathrm{~mm}$ and an SS-A of $>58 \mathrm{~mm}^{2}$ strongly predicted clinical evolution to the NAR cohort based on early MR imaging (Fig $3 A$ ).

There were considerably fewer patients with fetal MR imaging $(n=15)$, but the fraction of fetal patients in the NAR cohort $(n=10)$ was nearly identical to the fraction of neonatal patients in the NAR cohort ( $67 \%$ and $66 \%$, respectively). Mean gestational age was comparable in the NAR (34 \pm 3 weeks) and IT (33 \pm 5 weeks) cohorts. Both readers found a significant difference between cohorts in measurements of SS-MD, SS-P, and right Sig-

Table 3: Presence of VOGM morphology in early MR imaging scan data for neonatal at-risk and infant treatment cohorts

\begin{tabular}{lccc}
\hline \multicolumn{1}{c}{ VOGM Morphology } & Choroidal & Mural & Total \\
\hline Reviewer 1 & & & \\
Cohort & & & \\
$\quad$ NAR & 19 & 2 & 21 \\
$\quad$ IT & 9 & 2 & 11 \\
$\quad$ Total & 28 & 4 & 32 \\
$\chi^{2}(1, n=32)=0.495, P=.48$ & & & \\
Reviewer 2 & & & \\
$\quad$ Cohort & & & \\
$\quad$ NAR & 21 & 0 & 21 \\
IT & 4 & 7 & 11 \\
$\quad$ Total & 25 & 7 & 32 \\
$\chi^{2}(1, n=32)=17.105, P<.001$ & & & \\
$\kappa=0.48, P=.05$ & & & \\
\hline
\end{tabular}

Table 4: Presence of occipital sinus morphology in early MR imaging scan data for neonatal at-risk and infant treatment cohorts

\begin{tabular}{|c|c|c|c|}
\hline Presence of Occipital Sinus & No & Yes & Total \\
\hline \multicolumn{4}{|l|}{ Reviewer 1} \\
\hline \multicolumn{4}{|l|}{ Cohort } \\
\hline NAR & 0 & 21 & 21 \\
\hline IT & 6 & 5 & 11 \\
\hline Total & 6 & 26 & 32 \\
\hline \multicolumn{4}{|l|}{$\chi^{2}(1, n=32)=14.1, P<.001$} \\
\hline \multicolumn{4}{|l|}{ Reviewer 2} \\
\hline \multicolumn{4}{|l|}{ Cohort } \\
\hline NAR & 0 & 21 & 21 \\
\hline IT & 4 & 7 & 11 \\
\hline Total & 4 & 28 & 32 \\
\hline $\begin{array}{l}\chi^{2}(1, n=32)=8.3, P<.01 \\
\kappa=0.819, P<.001\end{array}$ & & & \\
\hline
\end{tabular}

Table 5: Cutoff values for NAR on fetal and neonatal MR imaging ${ }^{\mathrm{a}}$

\begin{tabular}{ccccccc}
\hline & AUC & $\mathbf{9 5 \%} \mathbf{C l}$ & $\boldsymbol{P}$ Value & $\begin{array}{l}\text { Cutoff } \\
\text { Value }\end{array}$ & Sensitivity & Specificity \\
\hline $\begin{array}{c}\text { Neonatal } \\
\text { SS-MD }\end{array}$ & 0.866 & $0.736-0.996$ & $<.001$ & $>6.2 \mathrm{~mm}^{2}$ & $86 \%$ & $82 \%$ \\
SS-A & 0.836 & $0.690-0.983$ & .002 & $>58 \mathrm{~mm}^{2}$ & $80 \%$ & $82 \%$ \\
Fetal scans & & & & & & \\
SS-MD & 0.980 & $0.919-1.000$ & .003 & $>5.2 \mathrm{~mm}^{2}$ & $90 \%$ & $100 \%$ \\
SS-A & 0.941 & $0.823-1.000$ & .007 & $>33 \mathrm{~mm}^{2}$ & $90 \%$ & $80 \%$ \\
\hline
\end{tabular}

${ }^{a}$ Probabilities determined by logistic regression, where straight or falcine sinus diameter on MR imaging was identified as a multivariable predictor of clinical outcome $(A \cup C=0.89, P<.001)$.
MD. There was no significant difference in measurements between cohorts for most varix measurements (varix mediolateral diameter, varix CC, and varix volume) for both readers. Here too, the interrater reliability was high $(>0.80)$ for most caliber measurements: varix mediolateral diameter, varix $\mathrm{CC}$, varix volume, SS-MD, SS-P, and left Sig-MD.

There were notably several missing parameters on fetal MR imaging, including BA, ICA bilaterally, and the sigmoid sinuses bilaterally. In most cases, the missing variables could not be measured due to suboptimal fetal positioning in all relevant slices. Only 1 fetal MR imaging had a missing SS-P value, due to the lack of a coronal sequence. Similarly, the presence of the occipital sinus and VOGM morphology was difficult to fully assess on the fetal MR imaging on a consistent basis.

Multivariable analysis again demonstrated that SS-MD $(\mathrm{AUC}=0.980, P=.003)$ and SS-A (AUC $=0.941, P=.007)$ were most predictive of clinical evolution to NAR (Table 5). By means of an ROC curve, the most predictive cutoff values for SS-MD and SS-A were $>5.2$ and $>33 \mathrm{~mm}^{2}$, respectively (Fig $3 B$ ).

Eleven patients had both an early neonatal and a fetal MR imaging, with an average time difference between these scans of $3.3 \pm 2.7$ weeks. If one compared the fetal with the neonatal scan in these cases, there was no significant difference in mean measurements of either the SS-MD $(P=.837)$ or SS-A $(P=.794)$ and the fetal and neonatal measurements were highly correlated for both SS-MD $(r=0.90, P<.001)$ and SS-A $(r=0.91, P<.001)$. High correlation between fetal and neonatal scans within the same patients suggests that there was a minimal differential change in the size of the SS-MD and SS-A measurements with time. Thus, data from the larger neonatal cohort could potentially be used to confirm the results in the much smaller fetal cohort.

As an illustration of the sharp increase in the likelihood of evolution to NAR with an increasing mediolateral diameter of the straight or falcine sinus, we calculated this probability, along with $95 \%$ confidence intervals, for a single reader (reader 2), as illustrated in Table 6.

\section{DISCUSSION}

Vein of Galen malformation is a rare congenital vascular anomaly with a complex and potentially devastating outcome. The presence of a cerebral arteriovenous shunt with resultant high cardiac output leads to multiple possible morbidities: severe pulmonary hypertension, increased cardiac preload, holodiastolic reversal of flow in the descending aorta with concomitant organ hypoperfusion, cerebral venous congestion and hypertension, and so forth. In severe cases, the persistent arteriovenous shunting may lead to multiorgan failure and progressive destruction of hemispheric white matter and cortical parenchyma. ${ }^{10}$ This severely affected cohort (NAR), representing approximately two-thirds of neonates with VOGM, continues to have a high mortality rate and a high rate of severe neurodevelopmental morbidity among survivors, despite advances in neuroendovascular management. ${ }^{7}$ However, other patients with VOGM of similar overall caliber do not decompensate physiologically, do not require intubation or cardiac 

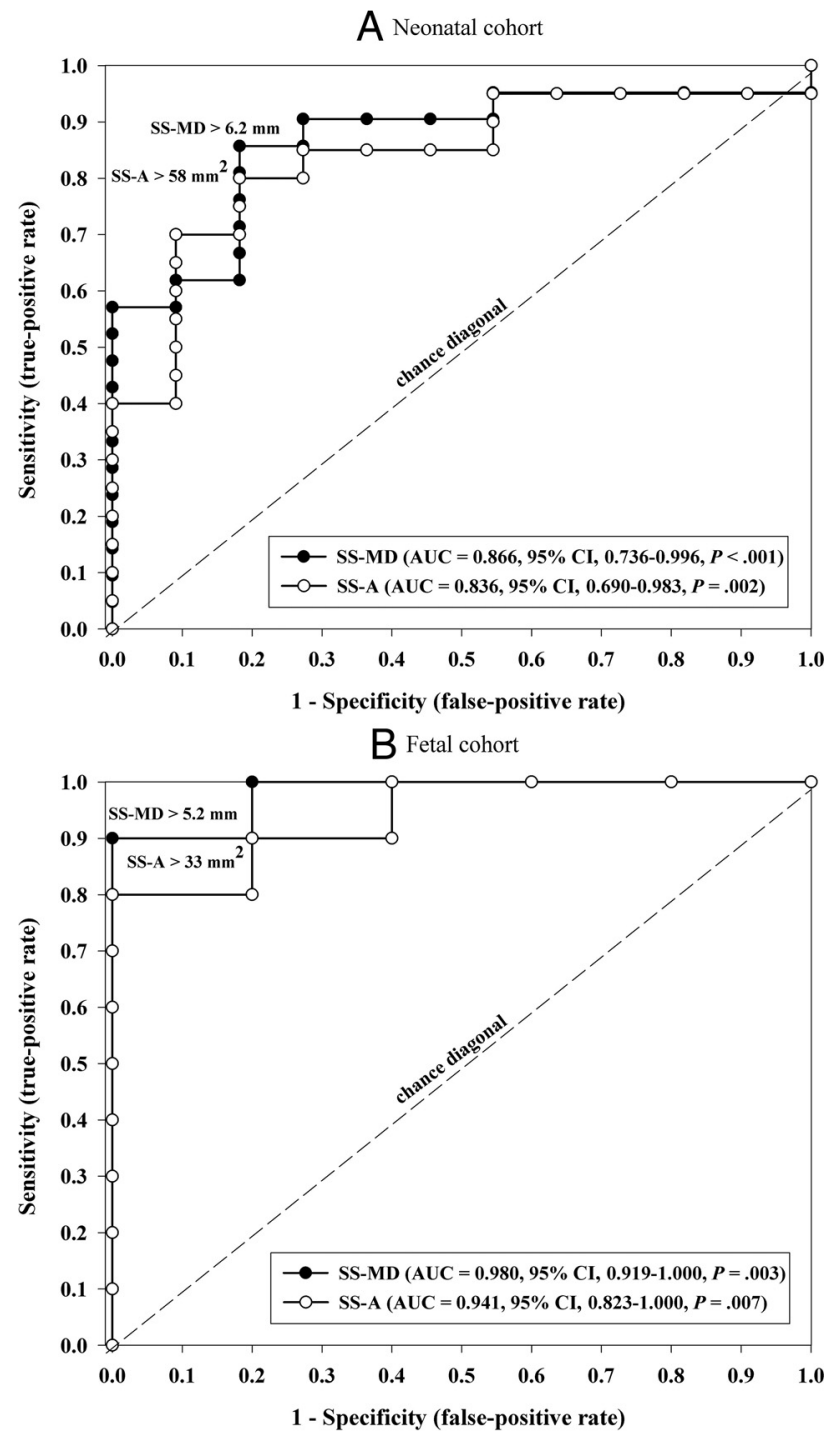

FIG 3. ROC curves showing measurements of the straight or falcine sinus area (SS-A) and the straight or falcine sinus maximal mediolateral diameter (SS-MD) at the shortest point of the sinus. Measurements from neonatal MR imaging $(A)$ and from fetal MR imaging $(B)$.

support, are discharged home from the neonatal intensive care unit, and are treated electively at several months of age. This latter group (IT) has a mortality rate of $\sim 10 \%$.

For the past 2 decades, the most widely used clinical scale for grading VOGM severity at presentation has been the Bicêtre neonatal evaluation score. ${ }^{11}$ Low scores on this scale $(<8)$, consistent with multiorgan presentation, have been used in many centers as a rationale for withholding all treatment efforts; high scores $(>12)$ have been used as a rationale for watchful waiting; and midrange scores (8-12), as an indicator of the need for urgent embolization. However, it has become clear that with expert treatment, some neonates scoring in the $<8$ range can have good outcomes and most referral centers no longer use strict score cutoffs for assignation of care. Moreover, the Bicêtre score is based on clinical criteria and is, thus, post hoc. It cannot be calculated during the antenatal period; and even at birth, it is impossible to predict how a given patient will evolve. Thus, to direct clinical resources appropriately, to offer families a realistic assessment of likely clinical outcome, and to identify an appropriate cohort to whom novel therapies should be directed, a need for a mechanism for prognosticating fetal outcomes exists. The present study was conducted to assess whether select vascular parameters on fetal MR imaging could play this role.

Measurements can be reliably obtained on MR imaging of most relevant fetal and neonatal vascular architecture. We found that vascular parameters on neonatal MR imaging revealed widespread dilation of both arterial and venous structures in patients in the NAR cohort compared with those in the IT cohort; to the best of our knowledge, these measurements have not been systematically evaluated previously.

However, we reasoned that the point of greatest constriction to flow return from the malformation to the systemic circulation might serve as the single most efficient predictor of neonatal outcome. Indeed, we found that in both fetal and neonatal MR imaging, patients with lesser constriction at this critical point (ie, those with a large mediolateral diameter or cross-sectional area of the straight or falcine sinus at its shortest section) were at significantly greater risk of evolving to NAR; thus, dilation at this point was sharply predictive of mortality and the need for neonatal intervention. The rationale behind choosing the mediolateral axis for diameter measurement was because the falcine and straight sinuses are tightly tacked by the dural reflection on the rostral and caudal edges of the sinus (thus the choice of the section with the shortest height as the section of interest), while the lateral walls of the sinus are free and unattached. This degree of freedom allows the sinus to expand or contract in the lateral plane as a function of flow; thus, the mediolateral diameter serves as an anatomic marker of flow. These measurements were readily obtained from both fetal and neonatal MR imaging and can be reliably quantified in either the axial or coronal plane, as long as a crossreferenced sagittal sequence is available to identify the shortest section of the sinus. While other studies have suggested broadly that a dilated straight sinus was a poor prognosticator for patients with VOGM on both MR imaging and sonography, none of these studies detailed where or how to measure dilation, and no quantitative or systematic analysis was performed. ${ }^{12,13}$ The specific measurement we put forward here has not been previously described. The utility of this technique in terms of readily differentiating cohorts with very low likelihood versus very high likelihood of evolution to NAR is illustrated in Table 6, even for a single MR imaging reader.

Most interesting, median prosencephalic varix size itself was not a predictor of clinical evolution to NAR versus IT, a finding in conflict with some earlier reports. Paladini et $\mathrm{al}^{12}$ found that a VOGM varix volume of $>20,000 \mathrm{~mm}^{3}$ was predictive of poor outcome and parenchymal injury. On the other hand, Saliou et $\mathrm{al}^{14}$ found that the size of the varix on prenatal MR imaging was not predictive of poor outcome, as others had reported. ${ }^{11}$ We would note that Paladini et al grouped patients with VOGM who had neurologic sequelae, neonatal death, and termination of pregnancy into a single cohort, while in the present study, NAR assignation was determined by neonatal death or need for urgent neonatal embolization. In the same study, Saliou et al reported on middle cerebral artery "pseudofeeders" on fetal MR imaging as a 
Table 6: Likelihood of clinical evolution to NAR as a function of the mediolateral diameter of the straight or falcine sinus at its shortest point ${ }^{\mathrm{a}}$

\begin{tabular}{lcc}
\hline $\begin{array}{c}\text { Straight or Falcine } \\
\text { Sinus Diameter }(\mathrm{mm})\end{array}$ & Probability of NAR & $\mathbf{9 5 \%} \mathrm{Cl}$ \\
\hline 0 & $4 \%$ & $1 \%-30 \%$ \\
1 & $8 \%$ & $2 \%-38 \%$ \\
2 & $14 \%$ & $3 \%-44 \%$ \\
3 & $24 \%$ & $8 \%-52 \%$ \\
4 & $38 \%$ & $18 \%-60 \%$ \\
5 & $53 \%$ & $33 \%-72 \%$ \\
6 & $68 \%$ & $49 \%-83 \%$ \\
7 & $80 \%$ & $60 \%-92 \%$ \\
8 & $88 \%$ & $69 \%-96 \%$ \\
9 & $93 \%$ & $75 \%-98 \%$ \\
10 & $96 \%$ & $80 \%-99 \%$ \\
11 & $98 \%$ & $84 \%-99 \%$ \\
12 & $99 \%$ & $87 \%-99 \%$ \\
13 & $100 \%$ & $90 \%-100 \%$ \\
\hline
\end{tabular}

a Probabilities determined by logistic regression, where straight or falcine sinus diameter on MR imaging was identified as a multivariable predictor of clinical outcome $(A \cup C=0.89, P<.001)$

risk factor for poor clinical prognosis, but we found making standardized assessments of middle cerebral artery branches in the Sylvian fissure to be very challenging, largely due to variable fetal positioning.

Quisling and Mickle ${ }^{15}$ performed transtorcular venous pressure measurements on a small cohort of patients. They found that patients with an enlarged straight or falcine sinus trended toward increased venous pressures. Additionally, 1 patient presented with a normal straight sinus and normal pressure; but across time, the straight sinus dilated and repeat measurement found elevated pressure. The relationship between straight or falcine sinus dilation and venous pressure is difficult to prove on the basis of that small study but does represent an interesting direction for further research.

The major limitation in this study is the small sample size (in keeping with the rarity of VOGM), along with incomplete measurements in some cases due to either image quality or lack of relevant MR imaging sequences. Even with these limitations, our central finding was quite robust.

\section{CONCLUSIONS}

We identified vascular anatomic characteristics on neonatal and fetal MR imaging that strongly predicted clinical outcome. Specifically, while the caliber of many arterial and venous structures statistically differed between the NAR and IT cohorts, the most robust differentiator was the width (mediolateral) and area of the straight or falcine sinus measured at the point of its shortest height (ie, the point of greatest constriction to flow return to the systemic circulation). This measurement clearly and unambiguously differentiated between high- and low-risk cohorts. The ability to accurately predict clinical evolution after birth based on fetal MR imaging can be of help for both caregivers and families, enabling better preparedness for urgent treatment and better planning for allocation of resources. Additionally, fetal patients who are very likely to evolve to NAR represent an appropriate cohort for novel therapeutic approaches.

\section{REFERENCES}

1. Recinos PF, Rahmathulla G, Pearl M, et al. Vein of Galen malformations: epidemiology, clinical presentations, management. Neurosurg Clin N Am 2012;23:165-77 CrossRef Medline

2. Raybaud CA, Strother CM, Hald JK. Aneurysms of the vein of Galen: embryonic considerations and anatomical features relating to the pathogenesis of the malformation. Neuroradiology 1989;31:109-28 CrossRef Medline

3. Bhattacharya JJ, Thammaroj J. Vein of Galen malformations. J Neurol Neurosurg Psychiatry 2003;74(Suppl 1):i42-44 CrossRef Medline

4. Brinjikji W, Krings T, Murad MH, et al. Endovascular treatment of vein of Galen malformations: a systematic review and meta-analysis. AJNR Am J Neuroradiol 2017;38:2308-14 CrossRef Medline

5. Yan J, Wen J, Gopaul R, et al. Outcome and complications of endovascular embolization for vein of Galen malformations: a systematic review and meta-analysis. J Neurosurg 2015;123:872-90 CrossRef Medline

6. Deloison B, Chalouhi GE, Sonigo P, et al. Hidden mortality of prenatally diagnosed vein of Galen aneurysmal malformation: retrospective study and review of the literature. Ultrasound Obstet Gynecol 2012;40:652-58 CrossRef Medline

7. Lecce F, Robertson F, Rennie A, et al. Cross-sectional study of a United Kingdom cohort of neonatal vein of Galen malformation. Ann Neurol 2018;84:547-55 CrossRef Medline

8. Gopalan V, Rennie A, Robertson F, et al. Presentation, course, and outcome of postneonatal presentations of vein of Galen malformation: a large, single-institution case series. Dev Med Child Neurol 2018;60:424-29 CrossRef Medline

9. Staffa SJ, Zurakowski D. Strategies in adjusting for multiple comparisons: a primer for pediatric surgeons. J Pediatr Surg 2020 Jan 23. [Epub ahead of print] CrossRef Medline

10. Alvarez H, Garcia Monaco R, Rodesch G, et al. Vein of Galen aneurysmal malformations. Neuroimaging Clin N Am 2007;17:189-206 CrossRef Medline

11. Lasjaunias PL, Chng SM, Sachet M, et al. The management of vein of Galen aneurysmal malformations. Neurosurgery 2006;59:(Suppl 3)S184-94; discussion S3-13 CrossRef Medline

12. Paladini D, Deloison B, Rossi A, et al. Vein of Galen aneurysmal malformation (VGAM) in the fetus: retrospective analysis of perinatal prognostic indicators in a two-center series of 49 cases. Ultrasound Obstet Gynecol 2017;50:192-99 CrossRef Medline

13. Yuval Y, Lerner A, Lipitz S, et al. Prenatal diagnosis of vein of Galen aneurysmal malformation: report of two cases with proposal for prognostic indices. Prenat Diagn 1997;17:972-77 Medline

14. Saliou G, Vraka I, Teglas JP, et al. Pseudofeeders on fetal magnetic resonance imaging predict outcome in vein of Galen malformations. Ann Neurol 2017;81:278-86 CrossRef Medline

15. Quisling RG, Mickle JP. Venous pressure measurements in vein of Galen aneurysms. AJNR Am J Neuroradiol 1989;10:411-17 Medline 